\title{
Gradientes de Textura com Linhas de Perspectiva e Horizonte Amplificam a Superestimação de Tamanho Relativo em Espaços Pictóricos
}

\author{
Texture Gradients with Perspective Lines and Horizon Amplify the \\ Overestimation of Relative Size in Pictorial Spaces
}

\author{
Nelson Torro-Alves* \& Sérgio Sheiji Fukusima \\ Universidade de São Paulo, Ribeirão Preto, Brasil
}

\begin{abstract}
Resumo
Comparações de tamanhos relativos em espaços representados pictoricamente foram investigadas sob influência de informações de profundidade. Quarenta participantes ajustaram, pelo método das escadas, os tamanhos de retângulos brancos no campo visual superior para serem percebidos como iguais aos tamanhos de cartas de baralho apresentadas no campo visual inferior. Durante a apresentação dos estímulos, informações pictóricas de profundidade eram disponibilizadas no fundo da tela do computador e os participantes foram solicitados a tipificar a estratégia utilizada para julgar os tamanhos percebidos. A linha do horizonte e o gradiente de linhas de perspectiva favoreceram a superestimação dos tamanhos percebidos em cerca de $8 \%$ e $12 \%$, respectivamente, e as comparações de tamanho foram coerentes com a constância de tamanho.

Palavras-chave: Tamanho relativo; linha do horizonte; percepção visual; tamanho familiar; indícios pictóricos de profundidade.
\end{abstract}

\begin{abstract}
Comparisons of relative size in pictorial spaces under the influence of depth cues were investigated. Using the staircase method, 40 participants adjusted the sizes of blank cards presented in the upper visual field to the size of playing cards presented in the lower visual field. During the presentation of the stimuli, pictorial depth cues were displayed in the computer screen and participants were asked to typify the strategy used to judge the perceived sizes. The horizon and the perspective line gradient contributed to the overestimation of the perceived size of blank cards in about 8 and $12 \%$, respectively, and size comparisons were coherent with the constancy of size.

Keywords: Relative size, horizon, visual perception, familiar size, pictorial depth cues.
\end{abstract}

A percepção visual do espaço tridimensional é determinada por diferentes tipos de informações ou indícios de profundidade (Gilliam, 1995). Os indícios musculares, por exemplo, fornecem informações de profundidade através dos processos de acomodação e convergência dos olhos. Os indícios binoculares de profundidade permitem que o observador extraia informações sobre a distância e o tamanho dos objetos em função da pequena diferença existente entre as imagens que chegam a cada um dos olhos. Os indícios monoculares de profundidade, por sua vez, apresentam um importante papel na apreensão do espaço tridimensional e são comumente utilizados nos processos de reconstrução da profundidade em fotografias, desenhos e representações pictóricas (Rock, 1985). Devido ao advento

\footnotetext{
* Endereço para correspondência: Universidade de São Paulo, Avenida Bandeirantes, 3900, Ribeirão Preto, SP, 14040-901. Fone: (16) 3602-4448. E-mail: nelsontorro@yahoo.com.br

Apoio Financeiro: $\mathrm{CNPq}$.

Este trabalho é parte da monografia de conclusão de curso do primeiro autor, defendida em 2001 no programa de Bacharelado Especial em Psicologia da Faculdade de Filosofia, Ciências e Letras de Ribeirão Preto - Universidade de São Paulo.
}

da construção de ambientes virtuais por técnicas de computação gráfica para simular espaços tridimensionais, torna-se importante compreender como as dimensões espaciais (tamanho e distância) dos objetos são determinadas nesses ambientes essencialmente pictóricos (Botella et al., 2004; Creem-Regehr, Willemsen, Gooch, \& Thompson, 2005; Dixon, Wraga, Proffitt \& Willians, 2000; Loomis \& Blascovich, 1999; Loomis \& Knaap, 2003).

Atualmente, muitos trabalhos têm investigado em especial o papel de alguns dos indícios monoculares de profundidade, tal como a linha do horizonte, o tamanho familiar e o gradiente de textura sobre a percepção de tamanho relativo. Gibson (1979) postulou que a percepção de tamanho e distância relativa é especificada por meio de relações dos objetos com o horizonte. De acordo com sua teoria, o horizonte é definido como o limite mais distante no plano do solo, tendo sua posição determinada pela altura do olho do observador. Assim, num ambiente natural, a distância da base de um objeto até o ponto onde este é interceptado pelo horizonte será correspondente à altura do ponto de observação (nível dos olhos do observador). Em uma representação pictórica (desenhos ou figuras), a altura do 
ponto de observação pode não ser conhecida, entretanto, se houver dois ou mais objetos em cena o tamanho relativo dos objetos será especificado por meio de relações de razão de horizonte. A razão de horizonte corresponde à razão do tamanho total pelo tamanho parcial do objeto que fica abaixo da linha do horizonte (Figura 1). Alguns trabalhos (Rogers, 1996) mostram que relações de razão de horizonte representam uma poderosa fonte de informação na determinação do tamanho relativo de objetos em representações pictóricas.

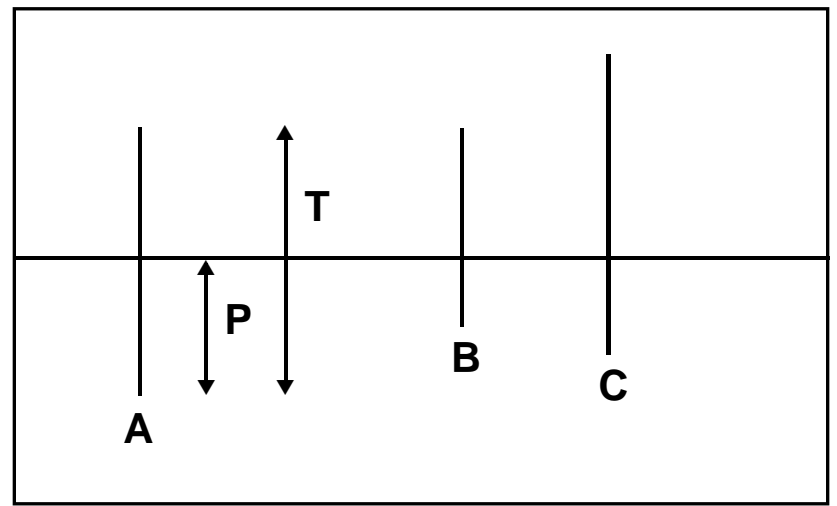

Figura 1. A razão de horizonte é definida por $\mathrm{T} / \mathrm{P}$, onde $\mathrm{T}$ corresponde ao tamanho total do objeto e $\mathrm{P}$ ao tamanho parcial do objeto que fica abaixo da linha do horizonte. O objeto A tem duas vezes a altura do ponto de observação (razão de horizonte =2). Os objetos B e C têm três vezes a altura do ponto de observação (razão de horizonte $=3$ ). Conseqüentemente, os objetos B e C têm uma vez e meia a altura do objeto A (baseado em Rogers, 1996).

Outra variável relevante para o estudo da percepção de tamanho é o tamanho familiar ou tamanho conhecido do objeto. Duas teorias principais explicam as relações existentes entre o tamanho familiar e sua influência nos julgamentos de tamanho e distância. A Teoria das Percepções de Tamanhos Anormais (Gogel \& Da Silva, 1987b) afirma que, ao menos em condições de reduzida estimulação visual (condições sob visão monocular e ausência de pistas de profundidade), a distância percebida de um objeto familiar é determinada pela distância de referência egocêntrica (DRE) do observador. Por meio da distância de referência egocêntrica, um objeto será percebido em um determinado ponto no espaço e o seu tamanho inferido de acordo com os princípios da hipótese da invariância entre tamanho e distância. O valor particular da distância de referência egocêntrica presente em uma determinada situação é dependente da tendência de distância especifica do observador para localizar o objeto no espaço. Assim, um objeto de tamanho normal será julgado de tamanho normal pelo observador somente se sua distância percebida, determinada pela distância de referência egocêntrica, corresponder a sua distância física. Se um objeto for apresentado aquém ou além da DRE do observador, seu tamanho percebido será menor ou maior, respectivamente, que seu tamanho real.

A outra teoria que estuda os efeitos do tamanho familiar sobre a percepção de tamanho e distância é a teoria do tamanho familiar. Esta teoria afirma que o tamanho familiar tem grande influência sobre o tamanho percebido e dividese em duas versões: a versão forte e a versão fraca (Predebon, 1994). Segundo a versão forte, o tamanho familiar é completamente efetivo, ou seja, um objeto familiar fisicamente de tamanho anormal aparentará sempre ter o tamanho de um objeto normal e será localizado perceptualmente para a distância que um objeto de tamanho normal estaria localizado. A teoria prediz que o ângulo visual terá pouca ou nenhuma influência no padrão das respostas de tamanho. A versão fraca admite que as pistas residuais de distância (acomodação e convergência) são propícias para se atenuar os efeitos do tamanho familiar. Na determinação do tamanho percebido duas forças potenciais estarão agindo em conflito; as pistas residuais de distância e as pistas do tamanho familiar. Deste modo, o tamanho percebido de um objeto anormal irá se aproximar do tamanho do objeto normal, sendo o tamanho da regressão diretamente proporcional à efetividade do tamanho familiar. Quanto maior a efetividade do tamanho familiar, mais próximo do tamanho normal o objeto será julgado.

A percepção de tamanho relativo pode ser ainda determinada por outras variáveis do arranjo óptico, tais como as características do gradiente de textura e do plano de fundo de figuras. Muitas das distorções de tamanho encontradas em ilusões visuais são decorrentes da interação entre os objetos da figura e o gradiente de textura em que foram inseridos (Williams \& Enns, 1996). As características do gradiente de textura podem também afetar a procura visual pelo tamanho dos objetos em representações pictóricas (Aks \& Enns, 1996).

Além dos efeitos perceptivos dos indícios pictóricos de profundidade, os julgamentos de tamanho podem ser também influenciados pelo tipo de atitude de julgamento do observador em relação ao estímulo visual. Em condições de reduzida estimulação visual, existem duas diferentes formas de avaliação dos estímulos. A atitude de julgamento objetivo toma como parâmetro o tamanho real do objeto, isto é, o tamanho que o objeto teria se pudesse ser medido com uma régua ou fita métrica. A atitude de julgamento aparente, por outro lado, tem como referência o tamanho aparente do objeto, ou seja, o tamanho que ele aparenta possuir (Predebon, 1992). Alguns trabalhos mostram que ao menos sob condições de reduzida estimulação visual estas instruções influenciam as estimativas de tamanho dos objetos de um modo distinto (Fukusima \& Da Silva, 1999; Predebon, 1992).

Apesar de já existirem estudos indicando o relevante papel dos indícios monoculares na percepção de profundidade, ainda há poucos trabalhos que tenham investigado simultaneamente o efeito destas variáveis em representações pictóricas. Por esta razão, o presente estudo teve por objetivo investigar os efeitos da linha do horizonte e de diferentes gradientes de textura sobre a percepção de tamanho relativo em representações pictóricas do espaço. Além disso, foram também investigadas as estratégias de julgamento utilizadas pelos observadores nas estimativas do tamanho relativo dos estímulos. 


\section{Participantes}

Método

Participaram voluntariamente quarenta universitários (19 homens, 21 mulheres) do campus da Universidade de São Paulo em Ribeirão Preto, com idade entre 17 e 30 anos e acuidade visual normal (6/6) ou superior em ambos os olhos com ou sem o uso de lentes corretivas. Os participantes foram distribuídos aleatoriamente em quatro grupos eqüitativos. Todos assinaram o termo de consentimento aprovado pelo Comitê de Ética da Faculdade de Filosofia, Ciência e Letras de Ribeirão Preto - Universidade de São Paulo (FCLRP-USP) e receberam ajuda de custo para ressarcir eventuais gastos para participar deste experimento.

\section{Material e Equipamento}

Um aparelho orthorather Bausch \& Lomb foi usado para medir a acuidade visual dos participantes. Um apoio para queixo posicionava a cabeça do participante a frente de um monitor (Philips, 21 ", modelo Brilliance) conectado a um computador 486DX2, $50 \mathrm{MHz}, 4 \mathrm{Mb}$ RAM, por meio de uma placa de vídeo Diamond SpeedStar24 de 1 Mb RAM.
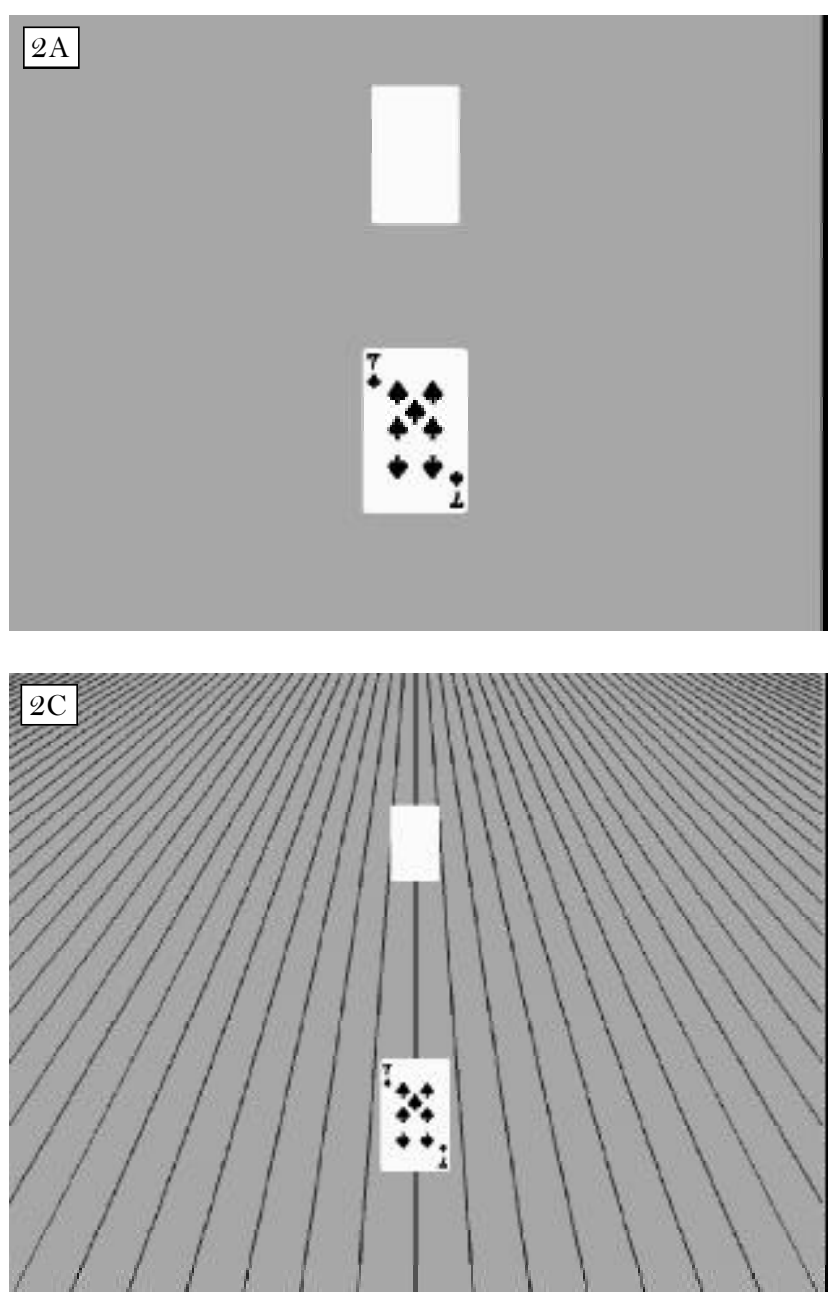

Um programa desenvolvido em Turbo Pascal 7.0 foi utilizado para gerar e apresentar os estímulos na tela do computador e para coletar as respostas dos participantes. O estímulo padrão era a figura de uma carta de baralho na parte inferior da tela e o estímulo teste era um retângulo branco na parte superior. A carta de baralho podia medir 5,4 x 3,5 cm (ver Figuras $2 \mathrm{~A}$ e $2 \mathrm{~B}$ ) ou $3,6 \times 2,3 \mathrm{~cm}$ (ver Figuras $2 \mathrm{C}$ e $2 \mathrm{D}$ ) e o tamanho inicial do retângulo branco correspondia a $130 \%$ ou $70 \%$ do tamanho das cartas de baralho. Além disso, os estímulos podiam ser apresentados em quatro condições de fundo de tela: (a) sem textura (Figura $2 \mathrm{~A}$ ), condição para o 1 - grupo de participantes; (b) gradiente de compressão de linhas horizontais (Figura 2B), para o 2 ㅇ grupo de participantes; (c) gradiente de linhas de perspectiva (Figura $2 \mathrm{C}$ ), para o $3{ }^{\circ}$ grupo de participantes e (d) gradiente combinado por compressão de linhas horizontais e linhas de perspectiva (Figura $2 \mathrm{D}$ ), para o $4^{\circ}$ grupo de participantes. Em cada uma das condições de fundo de tela podia-se também apresentar os estímulos sem o horizonte (como ilustrado na Figuras $2 \mathrm{~A}$ e $2 \mathrm{C}$ ) ou com horizonte localizado a $4 / 5$ da altura da tela do monitor (como ilustrado na Figuras $2 \mathrm{~B}$ e $2 \mathrm{D}$ ).
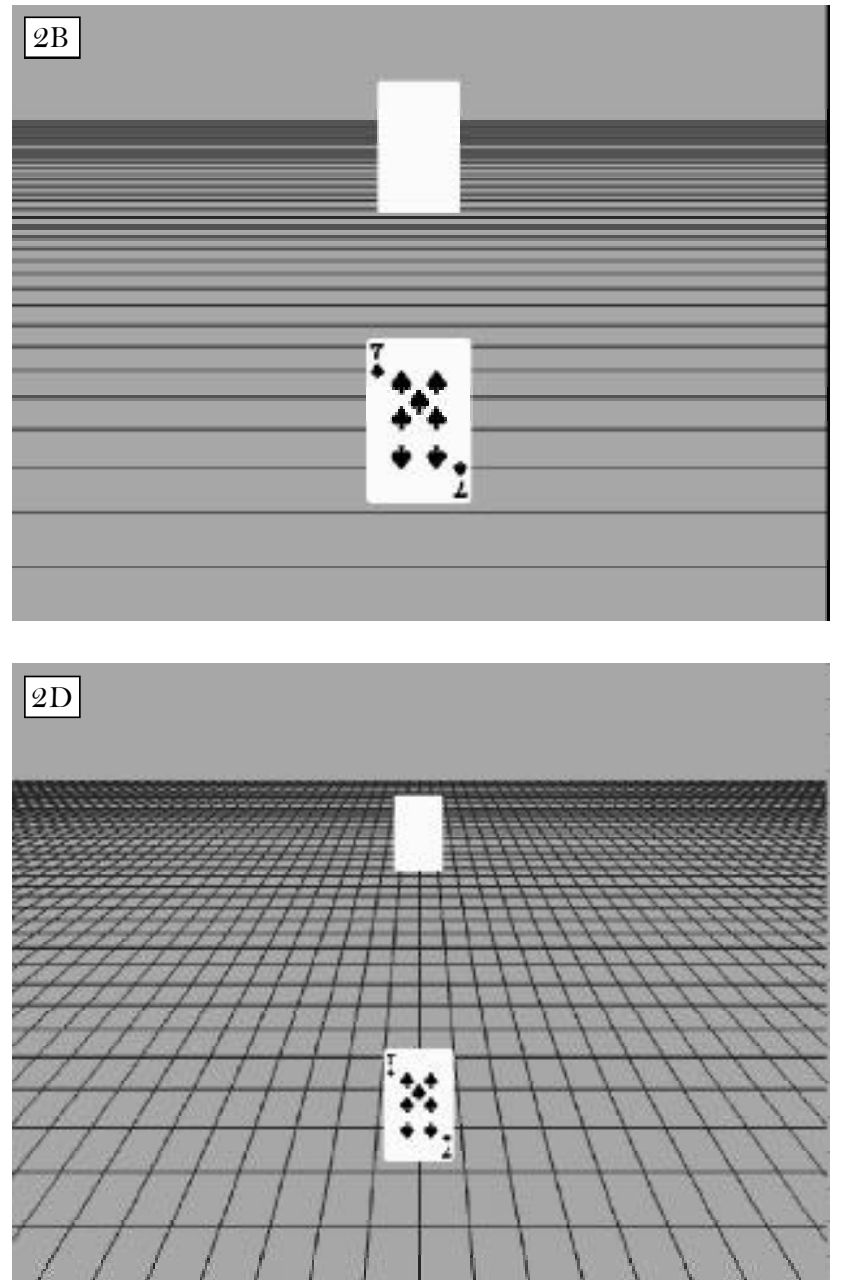

Figura 2. Exemplos de estímulos: (A) Ausência de textura e ausência do horizonte; (B) Gradiente de compressão e presença do horizonte; (C) Gradiente de perspectiva e ausência do horizonte; (D) Gradiente de compressão e perspectiva e presença do horizonte. 


\section{Procedimento}

Antes de iniciar a sessão, o participante com o olho esquerdo vendado se sentava à frente da tela do monitor e posicionava sua cabeça no apoio para o queixo de modo que o seu olho direito ficasse a $50 \mathrm{~cm}$ de distância do centro da tela do computador (ponto de fixação). A sessão era composta por quatro blocos, cuja ordem de execução foi aleatória: No bloco 1, comparava-se o tamanho do retângulo branco com o tamanho da carta de baralho menor na condição sem o horizonte; no bloco 2, a comparação de tamanhos com a carta de baralho menor era feita com o horizonte presente. No bloco 3, comparava-se o tamanho do retângulo branco com o tamanho da carta de baralho maior sem o horizonte e no bloco 4 a comparação de tamanho com a carta de baralho maior era feita com o horizonte presente.

Cada bloco foi constituído por apresentações simultâneas da carta de baralho e do retângulo branco pelo método das escadas duplas (Cornsweet, 1962). Neste método, havia uma série ascendente em que o tamanho inicial do retângulo branco era menor que o tamanho da carta de baralho e uma série descendente em que o tamanho inicial do retângulo branco era maior que o da carta de baralho. As apresentações dos estímulos de ambas séries eram intercaladas aleatoriamente ao longo da execução do bloco, de modo que o observador não podia prever a qual série elas pertenciam. Em cada apresentação, era primeiro mostrado uma tela cinza com o ponto de fixação por $500 \mathrm{~ms}$ e logo em seguida a tela com os estímulos (sem o ponto de fixação), na condição especificada de gradiente de textura, até que o participante emitisse uma resposta que indicasse qual dos estímulos era maior. Se o observador julgasse que o retângulo branco era maior que a carta de baralho deveria pressionar a letra "A" do teclado. Caso julgasse maior a carta de baralho, deveria pressionar a letra "D”. Essas respostas determinavam que o tamanho do retângulo branco na próxima apresentação da respectiva série deveria ser diminuído (resposta "A") ou aumentado (resposta "D") em $2 \%$ do tamanho da carta de baralho. Cada bloco se finalizava após 50 apresentações (25 apresentações da série ascendente e 25 da série descendente). Este número de apresentações foi previamente estabelecido em estudo piloto de modo que fosse suficiente para que os tamanhos ajustados dos retângulos brancos em ambas séries convergissem e se estabilizassem em torno do ponto de igualdade subjetiva (PIS) nas últimas dez apresentações de cada bloco.

Após a sessão, explicava-se aos participantes que as pessoas podem julgar os tamanhos dos estímulos considerando duas estratégias. A primeira seria a estratégia objetiva, isto é, os julgamentos dos tamanhos dos estímulos seriam baseados nas medidas físicas dos estímulos como se medidos por uma régua, desconsiderando assim as pistas de profundidade disponíveis nos gradientes de textura na tela do computador. A segunda seria a estratégia aparente que levaria em consideração os efeitos das pistas de profundidade dos gradientes de texturas apresentadas na tela do computador ao se julgar os tamanhos. Além disso, explicou-se também que as pessoas podem combinar as estratégias, alternando-se entre a estratégia objetiva e a estratégia aparente ou integrando simultaneamente ambas. Após os experimentadores se certificarem que os participantes haviam entendido corretamente os conceitos das estratégias de julgamento, eles eram indagados a relatar que estratégia foi utilizada para comparar os tamanhos dos estímulos durante o experimento. Em função do relato, os julgamentos foram classificados em julgamento objetivo, aparente ou uma combinação das estratégias.

\section{Análise dos Dados}

Os pontos de igualdade subjetiva (PIS) dos participantes em cada bloco foram estimados pelas médias dos tamanhos ajustados dos retângulos brancos nas últimas apresentações após notar sua convergência e estabilização. Este PIS foi em seguida utilizado para calcular o erro relativo pela fórmula:

$$
\operatorname{Erro}(\%)=100 *(\text { EP-PIS }) / E P
$$

em que Erro (\%) é o erro relativo e EP é o tamanho do estímulo padrão.

Erro relativo negativo indica que o estímulo teste foi ajustado maior que o estímulo padrão, ou seja, o estímulo teste foi subestimado perceptivelmente em relação ao padrão. Erro positivo indica que o estímulo teste foi ajustado menor que o padrão, ou seja, o teste foi superestimado perceptivelmente em relação ao estímulo padrão.

Estes erros relativos foram submetidos a uma ANOVA de três fatores, [quatro condições de textura $\mathrm{x}$ (dois tamanhos de carta de baralho $\mathrm{x}$ duas condições de horizonte)], sendo o fator "condição de textura" de medidas independentes e os fatores "tamanho da carta de baralho" e "condição do horizonte" de medidas correlacionadas (repetidas).

\section{Resultados}

A ANOVA indicou efeitos significativos dos fatores principais, mas sem interações entre eles $(p>0,05)$. Assim, as médias dos erros relativos agrupados somente em função desses fatores foram sumariadas nas Figuras 3 e 4. Nota-se na Figura 3 que os erros relativos ao se ajustar o tamanho do retângulo branco em relação à carta de baralho pequena foram maiores que aqueles em relação à carta de baralho grande $[F(1,36)=6,09 ; p<0,05]$, e que esses erros relativos também são maiores na presença do horizonte do que na sua ausência. $[F(1,36)=17,42 ; p<0,001]$. Isto mostra que reduzir o tamanho angular de um objeto familiar no campo visual inferior e apresentar o horizonte acima do nível dos olhos em condições pictóricas contribuem para a superestimação do tamanho relativo do retângulo branco apresentado acima do estímulo padrão. Na Figura 4 notase que as condições de textura afetaram os erros relativos $[F(3,36)=3,84 ; p<0,05]$ e que, mesmo na condição sem gradiente de textura, há uma tendência de se superestimar o retângulo branco localizado acima do ponto de fixação 
em relação a carta de baralho no campo visual inferior, o que indica naturalmente que a percepção de tamanho não é uniforme em toda a extensão do campo visual. Além disso, comparações por contraste indicaram que os erros relativos da condição sem textura e da condição de gradiente de compressão não se diferem entre si $[F(1,36)<1]$ e que os erros relativos da condição de gradiente de perspectiva e da condição combinada entre gradiente de compressão e perspectiva também não $[F(1,36)<1]$. Porém, nota-se que a média dos erros das condições envolvidas na segunda comparação é maior que a média dos erros das condições envolvidas na primeira $[F(1,36)=11,05 ; p<0,01]$, o que indica que o gradiente de compressão é ineficaz para afetar a percepção de tamanho e que somente o gradiente de perspectiva, mesmo ao ser combinado com o gradiente de compressão, é capaz de causar julgamentos superestimados do tamanho relativo do retângulo branco nas condições especificadas.

Os relatos das estratégias para julgar os tamanhos foram utilizados para reagrupar os erros relativos. As médias dos erros relativos e respectivos erros padrão e freqüências deste reagrupamento de cada condição de pista pictórica de profundidade foram sumariados na Figura 5. Nota-se que os erros relativos médios envolvendo estratégias objetivas variaram entre 3 e $5 \%$ e são muito menores que aqueles provenientes de estratégia aparente, cujos erros relativos médios variaram entre 10 e $15 \%$. Nota-se também que erros relativos médios das pessoas que utilizaram ambas estratégias variaram entre 7 e $13 \%$, valores intermediários entre ambas estratégias, mas muito mais próximos aos dos julgamentos de estratégias aparentes que objetivas.

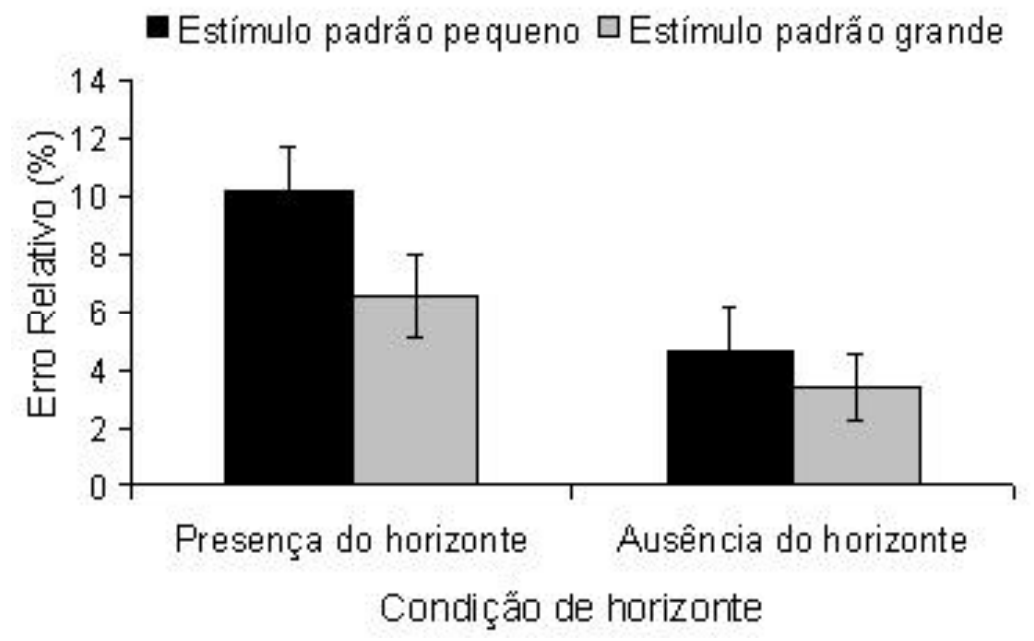

Figura 3. Efeito do horizonte sobre os julgamentos de tamanho relativo.

Nota. ${ }^{*}(p<0,001)$ presença do horizonte comparado com ausência do horizonte.

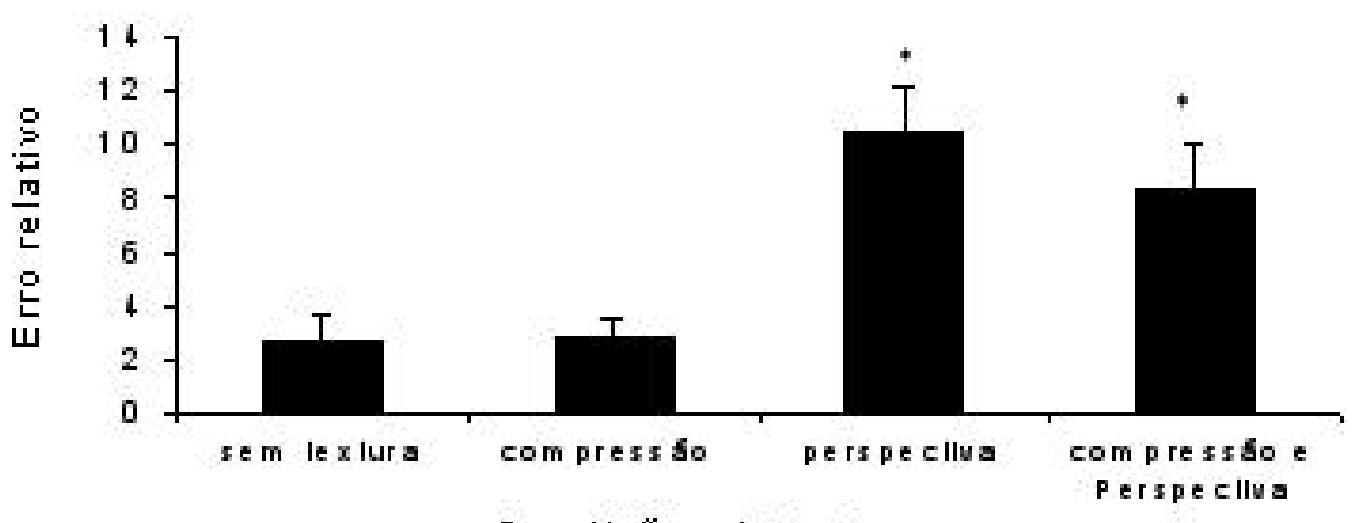

Condiçốes de textura

Figura 4. Efeitos da textura sobre os julgamentos de tamanho relativo.

Nota. ${ }^{*}(p<0,05)$ gradiente de perspectiva e gradiente de compressão e perspectiva comparados com a condição sem textura e com o gradiente de compressão. 


\section{$\square$ Julgamento objetwo $\square$ Julgamento objetivo e aparente $\square$ Julgamento aparente}

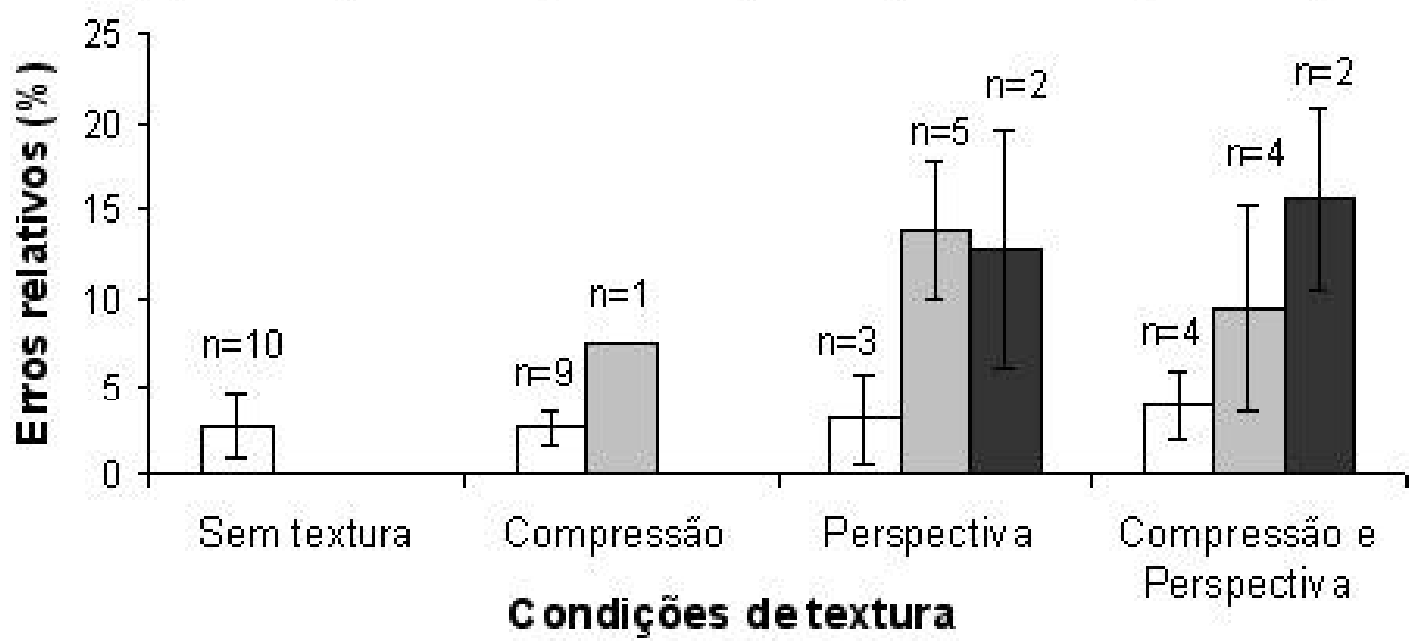

Figura 5. Erros relativos dos quatro grupos experimentais (condições de textura), distribuídos em função da estratégia de julgamento adotada pelos observadores no experimento ( $n=$ número de observadores).

Além disso, nota-se que as estratégias objetivas persistem em todas as condições de pistas pictóricas de profundidade, porém, predominante na condição controle (sem pista pictórica de profundidade) e na condição de gradiente de textura formada por linhas de compressão.

A estratégia aparente foi relatada na maioria dos casos combinada com a estratégia objetiva em todas as condições em que informações pictóricas de profundidade estavam disponíveis. E à medida que estas informações pictóricas de profundidade se tornam mais efetivas ou salientes, ela pode ser relatada eventualmente como estratégia exclusiva, como ocorreu nas condições em que havia linhas de perspectiva. Estes resultados sugerem que a estratégia objetiva tende a ser a estratégia básica para se julgar tamanhos pictóricos e à medida que informações de profundidade se tornam mais salientes, como ocorre com as linhas de perspectivas, a estratégia aparente tende a exercer um efeito maior nos julgamentos de tamanho pictóricos.

\section{Discussão}

Diferentemente de alguns trabalhos (Aks \& Enns, 1996; Bennett \& Warren, 2002; Found \& Muller, 2001) que investigaram o tempo de reação para mostrar o efeito de informações pictóricas de profundidade na percepção de tamanho, este estudo mostrou que o gradiente de textura com linhas de perspectiva e o horizonte são as principais informações de profundidade em espaços pictóricos que levam as pessoas a realçar a superestimação de tamanhos percebidos de objetos não familiares no hemicampo visual superior em relação a objetos familiares no hemicampo visual inferior.

Isto se demonstrou pela inexistência de efeito do gradiente de textura de compressão de linhas horizontais em relação à condição sem textura no tamanho percebido do retângulo branco e pelo aumento do erro em superestimar o tamanho do retângulo branco para 8 a 10\% nas condições em que as linhas de perspectiva eram componentes do gradiente de textura. Estes dois fatos sugerem que somente as linhas de perspectiva, em contraste à compressão de linhas horizontais, são efetivas para alterar o tamanho percebido.

Estes fatos também sugerem que gradientes de perspectiva linear são mais efetivos que os gradientes de compressão de linhas horizontais como informação primordial na percepção visual do espaço tridimensional, como já sugerido por Cutting e Millard (1984) e Goodenough e Gilliam (1997) em experimentos psicofísicos; e são também congruentes com pesquisas eletrofisiológicas em macacos e de ressonância magnética funcional em humanos (Sakata, Tsutsui \& Taira, 2005; Tsutsui, Taira \& Sakata, 2005), que evidenciaram o envolvimento de áreas cerebrais no lobo parietal e no sulco intra-parietal caudal no processamento de orientação no espaço tridimensional em presença de gradiente combinado de compressão de linhas horizontais e de perspectiva.

A pesquisa também mostrou que existe uma natural superestimação de aproximadamente $3 \%$ do tamanho do retângulo branco em condição sem informações pictóricas de profundidade; o que indica que a percepção de tamanho é anisotrópica, isto é, objetos de mesmo ângulo visual em locais diferentes do campo visual podem ser percebidos como de tamanhos diferentes. No caso, essa anisotropia se expressou entre a região superior e inferior do campo visual; o que é concordante com Fukusima e Faubert (2001), que mostraram que essa anisotropia manifesta-se tanto entre o hemicampo visual direito e esquerdo quanto entre o hemicampo visual superior e inferior. Essa anisotropia ainda não está explicada, mas uma plausível hipótese seria a distribuição ou alocação da atenção mais proeminente no campo visual superior em relação ao campo visual inferior e o seu 
hipotético efeito em amplificar o tamanho percebido. Ou ainda, ela seria uma tendência natural de acionar mecanismos de constância de tamanho, convergente com a idéia de Previc (1990) que o hemicampo visual superior é especializado em fornecer implicitamente relações espaciais em grandes distâncias, afastadas do observador, e o hemicampo visual inferior, especializado em fornecer relações espaciais em pequenas distâncias, próximas do observador.

Porém, independentemente das causas que levam a essa anisotropia, ela é realçada com a presença do horizonte no hemicampo visual superior, de modo que esta presença amplia muito mais os tamanhos percebidos de objetos desenhados no centro do campo visual em relação àqueles objetos desenhados afastados do horizonte. Isto confirma a relevância do horizonte em fornecer uma escala de tamanho aos objetos, como sugerido por alguns autores (Dixon et al., 2000; Rogers, 1996), e também revela sua contribuição em estabelecer as relações entre tamanhos e distâncias percebidas visualmente.

As representações pictóricas da carta de baralho, como fonte de tamanho familiar ou conhecido, em distâncias maiores e menores do observador (indicadas pelo tamanho pequeno e grande, respectivamente) também interferem na comparação de tamanhos, de maneira que, para o estímulo menor, a presença do horizonte aumenta a superestimação do tamanho percebido aproximadamente de $4 \%$ (sem o horizonte) para $10 \%$ em relação ao tamanho da carta de baralho; e para o estímulo maior, de aproximadamente 3,5\% (sem horizonte) para 6,5\% em relação ao tamanho da carta de baralho. Isto permite interpretar que essas informações pictóricas de profundidade e objetos de tamanho familiar fornecem também uma referência de escala de tamanho percebido, coerente com a constância de tamanho no espaço real (Holway \& Boring, 1941). Esta coerência com a constância de tamanho em condições pictóricas claramente se mostra nos tamanhos dos retângulos brancos ajustados menores que as cartas de baralho pela maioria dos observadores.

Porém de maneira menos clara, esta coerência com a constância de tamanho também é evidenciada ao se relacionar a estratégia aparente e a estratégia objetiva ao ajustar tamanhos com a disponibilidade e efetividade das informações de profundidade. A saber, entende-se neste experimento por estratégia aparente a ajustes de tamanhos do retângulo branco de maneira que o ângulo visual do retângulo branco fosse ajustado com base em sua inserção na distância representada no espaço pictórico, coerente com a constância de tamanho; e como estratégia objetiva, a ajustes de tamanho de modo que o ângulo visual do retângulo branco fosse ajustado igual ao da carta de baralho sem considerar as inserções dos objetos às distâncias representadas no espaço pictórico; e isto seria incoerente com a constância de tamanho (Miller, 2004).

Enquanto nas condições em que as informações de profundidade são menos efetivas (condição sem textura e gradiente de compressão) a estratégia objetiva é majoritária e a estratégia aparente é minoritária; e elas passam a mostrar tendência inversa (ou seja, a estratégia objetiva passa a ser minoritária e estratégia aparente passa ser majoritária) à medida que essas informações de profundidade são mais efetivas (gradiente de linhas de perspectiva e gradiente combinado por compressão de linhas horizontais e linhas de perspectiva). Nestas circunstâncias há um predomínio de relatos de que os julgamentos foram fundamentados conjuntamente em estratégias aparentes e objetivas, porém as magnitudes dos erros relativos desses julgamentos foram muitos similares aos julgamentos fundamentados somente em estratégias aparentes.

Essa contradição, entre estratégia relatada e estratégia efetuada na comparação de tamanhos pictóricos, pode ser interpretada como uma primazia em processar informações pictóricas disponíveis de profundidade que levam a constância de tamanho, indicando que este processo perceptual pode ser automático e anteceder a consciência dos observadores. Porém, salienta-se que relatos de estratégias de julgamento de tamanho são lábeis, pois o processo decisório em tipificar o julgamento pode ser afetado pelas instruções fornecidas aos observadores, pelas informações de profundidade disponíveis e por fatores cognitivos relativos à memória e a inferências sobre relações espaciais entre objetos (Alves \& Fukusima, 2005; Fukusima \& Da Silva, 1999; Gogel \& Da Silva, 1987a; Miller, 2004; Predebon, 1992).

Em suma, este estudo demonstrou que gradiente de textura formado por linhas de perspectiva e o horizonte são pistas pictóricas de profundidade que efetivamente distorcem o tamanho percebido de um retângulo branco no hemicampo visual superior em relação a um objeto de tamanho familiar no hemicampo visual inferior de modo coerente com o fenômeno da constância de tamanho. Porém, essa coerência não é revelada claramente quando os observadores são solicitados a relatar suas estratégias de julgamentos.

\section{Referências}

Aks, D. J., \& Enns, J. T. (1996). Visual search for size is influenced by a background texture gradient. Journal of Experimental Psychology: Human Perception and Performance, 22(6), 1467-1481.

Alves, N. T., \& Fukusima, S. S. (2005). Efeitos de instruções e do horizonte nos julgamentos de tamanhos relativos pictóricos. Psicologia: Teoria e Pesquisa, 21(2), 157-162.

Bennett, D., \& Warren, W. (2002). Size scaling: Retinal or environmental frame of reference. Perception \& Pschophysics, 64(3), 462-477.

Botella, C., Villa, H., Garcia-Palacios, A., Banos, R. M., Perpina, C., \& Alcaniz, M. (2004). Clinically significant virtual environments for the treatment of panic disorder and agoraphobia. Cyberpsychology and Behavior, 7(5), 527-535.

Cornsweet, T. (1962). The staircase method in psychophysics. American Journal of Psychology, 75, 485-491.

Creem-Regehr, S. H., Willemsen, P., Gooch, A. A., \& Thompson, W. B. (2005). The influence of restricted viewing conditions on egocentric distance perception: Implications for real and virtual indoor environments. Perception, 34(2), 191-204.

Cutting, J. E., \& Millard, R. T. (1984). Three gradients and the perception of flat and curved surfaces. Journal of Experimental Psychology: General, 113(2), 198-216. 
Dixon, M. W., Wraga, M., Proffitt, D. R., \& Willians, G. C. (2000). Eye height scaling of absolute size in immersive and nonimersive displays. Journal of Experimental Psychology: Human Perception and Performance, 26(2), 582-593.

Found, A., \& Müller, H. J. (2001). Efficient search for size targets on a background texture gradient: Is detection guided by discontinuities in the retinal-size gradient of items? Perception, $30(1), 21-48$.

Fukusima, S. S., \& Da Silva, J. A. (1999). Familiar size as a cue to size and distance estimates. In P. R. Killen \& W. R. Uttal (Eds.), Proceedings of the Twentieth Annual Meeting of the International Society for Psychophysics (pp.198-203). Tempe, AZ: International Society for Psychophysics.

Fukusima, S. S., \& Faubert, J. (2001). Perceived lenght in the central visual field, evidences for visual field asymmetries. Vision Research, 41(16), 2119-2126.

Gibson, J. J. (1979). The ecological approach to visual perception. Boston: Houghton Mifflin.

Gilliam, B. (1995). The perception of special layout from static optical information. In W. Epstein \& S. Rogers (Eds.), Handbook of perception and cognition: Perception of space and motion (pp. 23-67). London: Academic Press.

Gogel, W. C., \& Da Silva, J. A. (1987a). A two-process theory of the response to size and distance. Perception $\&$ Psychophysics, $41(3), 220-238$.

Gogel, W. C., \& Da Silva, J. A. (1987b). Familiar size and the theory of off-sized perceptions. Perception \& Psychophysics, $41(4), 318-328$.

Goodenough, B., \& Gilliam, B. (1997). Gradients as visual primitives. Journal of Experimental Psychology: Human Perception and Performance, 23(2), 370-387.

Holway, A. H., \& Boring, E. G. (1941). Determinants of apparent visual size with distance variant. American Journal of Psychology, 54, 21-37.

Loomis, J. M., \& Blascovich, J. J. (1999). Immersive virtual environment technology as a basic research tool in psychology. Behavior Research Methods, Instrument, E Computers, 31(4), 557-564.
Loomis, J. M., \& Knapp, J. M. (2003). Visual perception of egocentric distance in real and virtual environments. In L. J. Hettinger \& M. W. Haas (Eds.), Virtual and adaptative environment (pp. 21-46). Hillsdale, NJ: Erlbaum.

Miller, R. J. (2004). An empirical demostration of the interactive influence of distance and flatness information on size perception in pictures. Empirical Studies of the Arts, 22, 1-21.

Predebon, J. (1992). The role of instructions and familiar size in absolute judgments of size and distance. Perception $\Xi^{\circ}$ Psychophysics, 51(4), 344-354.

Predebon, J. (1994). Perceived size of familiar objects and the theory of off sized perceptions. Perception \& Psychophysics, 56(2), 238-247.

Previc, F. H. (1990). Functional specialization in the lower and upper visual fields in humans: Its ecological origins and neurophysiological implications. Behavioral and Brain Sciences, 13, 519-565.

Rock, I. (1985). La perception. Barcelona, España: Prensa Cientifica.

Rogers, S. (1996). The horizon-ratio relation as information for relative size in pictures. Perception $\&$ Psychophysics, 58(1), 142152 .

Sakata, H., Tsutsui, K., \& Taira, M. (2005). Toward an understanding of the neural processing for $3 \mathrm{D}$ shape perception. Neuropsychologia, 43(2), 151-161.

Tsutsui, K., Taira, M., \& Sakata, H. (2005). Neural mechanisms of three-dimensional vision. Neuroscience Research, 51(3), 221-229.

William, P. A., \& Enns, J. T. (1996). Pictorial depth and framing have independent effects on the horizontal-vertical illusion. Perception, 25(8), 921-926.
Recebido: 30/03/2005 $1^{a}$ revisão: $26 / 10 / 2005$

$2^{a}$ revisão: $25 / 05 / 2006$

Aceite final: 09/06/2006 